\title{
Medición automática de variables antropométricas para la evaluación de la respiración usando visión artificial
}

\section{Automatic measurement of anthropometric variables for the evaluation of respiration using artificial visión}

\author{
Héctor Marino-Vera ${ }^{1}$ \\ Luis Enrique Mendoza² \\ Oscar Eduardo Gualdrón-Guerrero ${ }^{3}$
}

Recibido: marzo 15 de 2017

Aceptado: junio 29 de 2017

\begin{abstract}
Resumen
La evaluación fonoaudiológica de la respiración es una herramienta indispensable para el profesional de la salud ya que permite conocer qué dificultades tiene la persona al respirar. Este protocolo en la actualidad se realiza de manera manual con ayuda de pocos marcadores, los cuales no permiten caracterizar la postura corporal, relacionada con distancias, ángulos y posiciones de manera general y segura. Este artículo presenta una novedosa alternativa en el desarrollo de un sistema para mediciones automáticas de variables antropométricas, que intervienen en la evaluación fonoaudiológica de la respiración, usando visión artificial. Se tomaron variables en los 4 planos: frontal, posterior, sagital izquierdo y sagital derecho; igualmente, se incluyeron 32 marcadores para obtener 35 variables antropométricas. El principio de funcionamiento del algoritmo automático para el reconocimiento de variables antropométricas, se probó con dos pacientes, logrando un porcentaje de error general del sistema de $2,81 \%$. Es importante resaltar que este desarrollo permitirá al especialista apoyarse en una herramienta digital para lograr medidas más precisas, mejorando la exactitud y la precisión en el diagnóstico.
\end{abstract}

Palabras clave: evaluación fonoaudiológica de la respiración, postura corporal, variables antropométricas, visión artificial.

\begin{abstract}
The speech-language evaluation of breathing is a tool important for the health professional because it allows to know the difficulties of people when breathing. This protocol is used manually with the help of a few markers, and not allows characterization of body posture, measuring to distances, angles and positions. This article presents a novel alternative in the development of a system for automatic measurements of anthropometric variables, which intervene in the phonoaudiological assessment of respiration, using artificial vision. Variables were taken on the 4 planes: frontal, posterior, left sagittal and right sagittal; Also, 32 markers were included to obtain 35 anthropometric variables. The operating principle of the automatic algorithm for the recognition of anthropometric variables was tested with two patients, achieving an overall system error rate of $2.81 \%$. It is important to emphasize that this development will allow the specialist to rely on a digital tool to achieve more precise measurements, improving accuracy in measurements and diagnosis.
\end{abstract}

Keywords: phonoaudiological evaluation of breathing, body posture, anthropometric variables, artificial vision.

1 Ingeniero en Telecomunicaciones, Universidad de Pamplona, Pamplona, Colombia. E-mail: hectorver@live.com 2 Ingeniero Electrónico, Magíster en Ingeniería Biomédica, Universidad de Pamplona, Pamplona, Colombia. E-mail: luis.mendoza@ unipamplona.edu.co

3 Ingeniero Electrónico, Doctor en Ingeniería Electrónica, Universidad de Pamplona, Pamplona, Colombia. E-mail: oscar.gualdron@ unipamplona.edu.co 


\section{Introducción}

El ser humano oxigena el cuerpo a través de la respiración, proceso que se ve afectado por alteraciones posturales y anomalías en la anatomía facial, entre otros problemas causados por la mala respiración (Orjuela-Juan de Dios et al., 2015). Además, la respiración se lleva a cabo, la mayoría de veces, sin que el sujeto tenga la mínima idea de lo que sucede. Es importante mencionar que un mal desarrollo del proceso de respiración en edades entre 3 y 5 años, puede llevar a tener posturas corporales anormales y por ende alterar las posturas corporales que se relacionan con la respiración, llevando a realizar esfuerzos inadecuados para compensar dicho proceso (Corvalan-Conta, 2013).

En la rama de la fonoaudiología se han creado protocolos para el análisis de la postura, para encontrar los posibles problemas que afectan al paciente. El más conocido actualmente es el llamado protocolo para evaluación fonoaudiológica de la respiración, desarrollado por Susanibar (2014). Existe software especializado en medidas de la postura como Adibas Posture y Body Posture, pero no cumplen a cabalidad con las medidas que requiere el protocolo. Estos programas son limitados y con poca capacidad de expansión de aplicaciones por parte del usuario (Porto de Freitas-Camelo et al., 2015).

La visión artificial presenta una alternativa para el reconocimiento de imágenes, habiendo sido aplicada en áreas tan disímiles como: determinación del grado de madurez de frutas, posicionamiento de herramientas, reconocimiento de rostros y gestos, sistemas de control de posición e identificación de rutas para la movilidad de robots, por mencionar algunas (Bonilla-González, \& Prieto-Ortiz, 2016; Cárdenas, \& Prieto-Ortiz, 2015; Cerón-Correa, Salazar-Jiménez, \& PrietoOrtíz, 2013; León-Medina, \& Torres-Barahona,
2016; Gutiérrez-Ríos, Martínez-Oviedo, \& PeñaCortés, 2013).

También se han desarrollado diversos trabajos usando visión artificial como punto de partida para la medición de variables antropométricas. Entre ellos se tienen los trabajos de: Tsoli et al. (2014), Gunay (2007), Baek y Lee (2012), Espitia-Contreras et al. (2014), y González et al. (2009), los cuales son muy relevantes en el análisis postural de la respiración; sin embargo, no incluyen la medición en tiempo real de las variables antropométricas de los 4 planos anatómicos, a saber: frontal, lateral izquierdo, lateral derecho y posterior. Estas propuestas no permiten tener una dimensión de alta resolución de las mediciones, de modo que el especialista no logra desarrollar diagnósticos efectivos y precisos en todos los planos.

En la actualidad no existe un software que permita medir todas las variables antropométricas relacionadas con los protocolos de la respiración, de manera automática y en tiempo real;esto implica que las mediciones que se realizan hoy en día sean subjetivas pues dependen de la pericia de quien las efectúe. La detección de malas prácticas de respiración permitirá en un futuro prevenir problemas tales como: cansancio, estrés, falta de concentración e hiperactividad, entre otras (Cruz-Mena, \& Moreno-Bolton, 2002; Villar, Jareño, \& Álvares, 2007). Además, desarrollar una herramienta tecnológica que le permita al especialista lograr de manera más rápida y precisa sus análisis, llevará a que se propongan nuevas alternativas de mejoramiento de la calidad de vida de las personas (Vargas et al., 2015).

En este artículo se presenta el desarrollo de un sistema automático basado en visión artificial, que permite la medición antropométrica en los cuatro planos anatómicos; esto con el fin de hallar todas las medidas que requiere el protocolo de medición fonoaudiológica de la respiración, además de facilitar y reducir tiempo en su implantación. 


\section{Marco teórico y metodología}

\subsection{Importancia de evaluar la respiración}

La respiración humana es un proceso de ventilación básicamente mecánico que renueva parcial y cíclicamente el aire alveolar, alternando los procesos de entrada de aire o inspiración y la salida del mismo o espiración, manteniendo dentro del pulmón una composición adecuada para el intercambio gaseoso (Cruz-Mena, \& Moreno-Bolton, 2002). El proceso de respiración está relacionado con: el abdomen, el esternón, la espalda, los músculos y cartílagos que intervienen en la fonación, junto con el diafragmáticoabdominal (Figueredo-Ruiz, \& Castillo-Martínez, 2016). Lo anterior indica la importancia de realizar el análisis del proceso respiratorio, ya que permite relacionar problemas de respiración con problemas fisiológicos, los cuales pueden ser corregidos si se detectan a tiempo, como ocurre con la mayoría de patologías en el ser humano (Hernández, Benjumea, \& Tuso, 2013; GelvezMunevar, Torres, Moreno-Muñoz, \& Bautista-Rojas, 2013).

En este proyecto el proceso manual de medición se realizó por un fisioterapeuta y un fonoaudiólogo, aplicando el protocolo de medición de Susanibar (2014). Las medidas se realizaron con una cinta métrica de precisión, Previa ubicación de los marcadores. Adicionalmente, se realizaron 3 mediciones por cada parámetro con el objetivo de obtener un promedio de las mismas. Todas las mediciones fueron tomadas desde el centroide de cada uno de los marcadores.

En la figura 1 se presenta el diagrama de bloques del sistema propuesto para la medición automática de variables antropométricas para la evaluación de la respiración usando visión artificial.

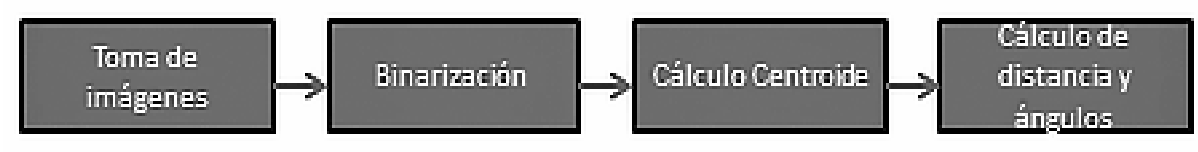

Figura 1. Diagrama en bloques del proceso.

El proceso llevado a cabo consta de 4 ciclos: el primero es la toma de imágenes basadas en un kinect, luego se realiza el proceso de binarización con el objetivo de tomar el centroide de cada marcador puestos en el paciente; en tercer lugar se realizan los cálculos de distancias y centroides, para terminar mostrando los resultados en la interfaz gráfica de usuario.

2.2 Segmentación de zonas y medición de variables antropométricas

La metodología se plantea en una serie de etapas basadas en segmentación, cálculos de distancias y cálculo de ángulos, usando técnicas de visión artificial (Bonnet, \& Venture, 2015; González, Woods, \& Eddins, 2009). Se tomaron imágenes de los cuatro segmentos de los planos antropométricos del sujeto. La resolución de la imagen es de $360 * 480$ pixeles. La figura 2 ilustra los 4 planos antropométricos tomados para realizar las medidas. 


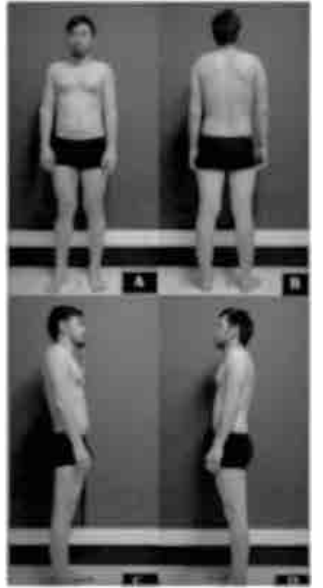

Figura 2. Imagen adquirida por el Kinect: A) plano Frontal, B) Plano posterior, C) Plano Sagital Izquierdo, D) Plano Sagital Derecho.

La segmentación del plano frontal se realiza con base en los marcadores antropométricos adheridos al cuerpo, que se ubican en la parte frontal. Se toma la imagen original y se descompone en 3 componentes, a saber: Rojo(R), Verde (G) y Azul (B). Utilizando los espacios de color se realizan restas entre las matrices, tal y como se indica en la ecuación 1.

$$
r=R-G, g=R-B, b=G-B
$$

Luego de la resta, se realiza una binarización por el método Otsu, como lo proponen Dixon y Leonardo (2013), donde T, es el umbral de binarización, tal como se indica en la ecuación 2.

$$
I_{1}(f, c)=\left\{\begin{array}{l}
1 \leftrightarrow I(f, c)>T \\
0 \leftrightarrow I(f, c) \leq T
\end{array}\right\}
$$

Después de realizar la binarización se procede a convertir la imagen a una representación de etiquetas, labels en inglés. Este proceso se refuerza con un filtrado por área para eliminar los ruidos (objetivos de áreas pequeñas). La ecuación 3 muestra el proceso matemático del filtrado por área. Nótese que todos los objetos que tienen áreas por debajo de $U$, se ponen en cero. En este caso el umbral seleccionado fue de 100.

$$
\left\{\begin{array}{l}
A_{i}<U \rightarrow O_{i}=0 \\
A_{i}>U \rightarrow O_{i}=O_{i}
\end{array}\right\}
$$

Donde $0 \rightarrow$ Objetos y $\mathrm{A}$ las áreas

$i=1,2,3 \ldots . N, N$ es el numero de objetos

En este trabajo las áreas de los objetos importantes contienen valores por encima de 100 pixeles, por ello el filtrado por área elimina áreas que estén por debajo de ese valor. Luego se obtiene la posición del centroide de cada región, obteniendo las coordenadas en las que se encuentra el marcador en la imagen digital. En este trabajo la forma de los marcadores se modela con formas geométricas, tal y como se ilustra en las ecuaciones 4 a 6 , que corresponden a las formas matemáticas para hallar los centroides en un círculo, triángulo y rectángulo, respectivamente.

$$
\begin{gathered}
C_{x}=C_{y}=\frac{1}{4} \pi r^{4} \circ C_{x}=\frac{D}{2}, C_{y}=\frac{D}{2} \\
C_{x}=C_{y}=\frac{1}{36} b h^{3}, C_{x}=\frac{b}{2} \quad C_{y}=\frac{h}{2} \\
C_{x}=\frac{b}{2}, C_{y}=\frac{h}{2}
\end{gathered}
$$

Con las coordenadas de cada centroide, y aplicando las ecuaciones 7 y 8 , correspondientes a la ecuación de la distancia y al teorema del coseno, respectivamente, se obtienen las mediciones antropométricas de cada plano. El plano frontal contiene 8 mediciones de longitud, el plano posterior contiene 8 longitudes; mientras que el plano sagital, tanto izquierdo como derecho, contiene 8 mediciones de longitudes y 4 ángulos faciales.

$$
\begin{gathered}
d C=\sqrt{\left(C x_{2}-C x_{1}\right)^{2}-\left(C y_{2}-C y_{1}\right)^{2}} \\
d C \rightarrow \text { Distancia entre centroides. } \\
C x_{N} \rightarrow \text { Centroide en } X . \\
C y_{N} \rightarrow \text { Centroide en } Y . \\
c^{2}=a^{2}+b^{2}-2 a b \cos (\varnothing)
\end{gathered}
$$




$$
\sigma=\cos ^{-1}\left(\frac{a^{2}+b^{2}-c^{2}}{2 a b}\right)
$$

\subsection{Metodología}

En el desarrollo del trabajo se utilizó un sistema kinect, el cual toma las imágenes que permiten realizar el estudio. Se utilizaron herramientas matemáticas como binarización, filtrado por área, restas de imágenes y obtención de propiedades, conocidas como centroide, en cada imagen. El desarrollo del algoritmo se realizó en matlab. Los resultados fueron analizados y comparados con los resultados del estudio manual, con el fin de verificar exactitud y corregir errores del sistema automático. Los resultados se obtuvieron midiendo las variables de distancia y ángulos en cada imagen tomada, una vez reconocidos los marcadores.

\section{Resultados y discusión}

La figura 3 muestra el resultado en el plano frontal para la detección de marcadores de brazo y pecho. Nótese cómo la binarización permite que solo los marcadores sean información importante y lo demás se considera información no importante; nótese también cómo los marcadores no tienen una forma geométrica regular definida y por esto toma importancia la búsqueda de la forma geométrica más adecuada para hallar el centroide. Los puntos de referencia para tomar las mediciones se representan de color blanco en la imagen binaria, en este caso 10 puntos.

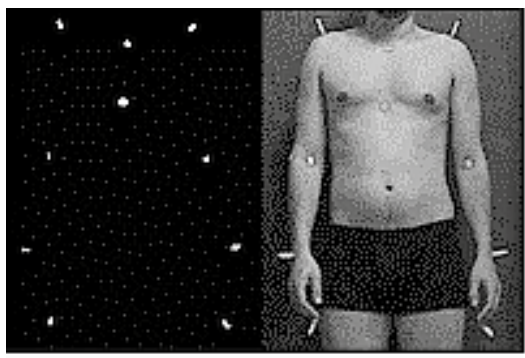

Figura 3. Binarización y eliminación de zonas de no interés.
La figura 4 muestra claramentela segmentación delas 4 zonas o planos sagitales, evidenciando la correcta segmentación de los planos. Nótese como se realizan interpolaciones lineales entre los diferentes puntos donde se encuentran los marcadores asignados en cada plano (líneas blancas).

Las figuras $4 c$ y $4 d$, muestran la segmentación de variables antropométricas del rostro del sujeto (líneas blancas) esto con el fin de obtener las medias faciales y ángulos para crear un perfil facial del mismo. Estas variables son esenciales en el proceso de respiración de cada sujeto. Es decir, cada sujeto puede tener dos patrones específicos en su respiración en cuanto a ángulos se refiere, lo que permitirá en un futuro mostrar los perfiles normales en sujetos sanos y a partir de ellos, buscar y detectar de manera automática problemas relacionados con la actividad respiratoria.

En la figura 4 semuestra un ejemplo de la toma de las imágenes y la ubicación de los marcadores, en este caso 2 sujetos diferentes en las 4 posiciones de estudio. Nótese como se trazan líneas para lograr realizar el cálculo de las distancias y así mismo estas líneas sirve de guía para encontrar el valor de los ángulos

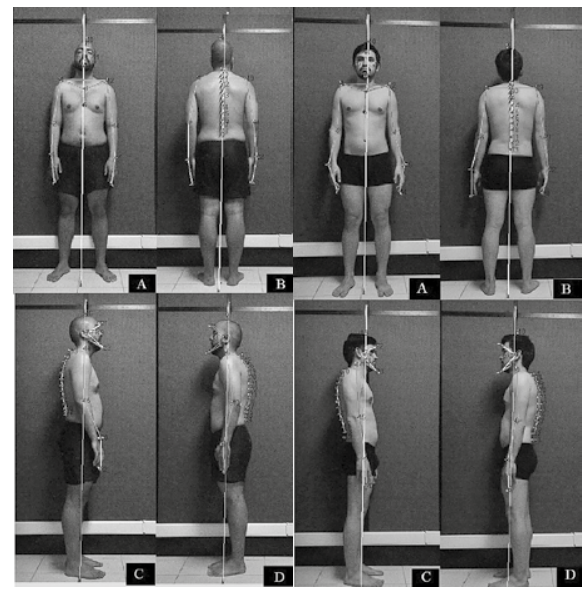

Figura 4. Imagen sujetos de prueba: A) plano Frontal, B) Plano Posterior, C) Plano Sagital Izquierdo, D) plano Sagital Derecho. 
El algoritmo se probó con dos sujetos, con los resultados que se muestran en las tablas 1 y 2 . Los valores de referencia corresponden a las mediciones tomadas manualmente por un fonoaudiólogo. Las tablas muestran los porcentajes de error de las mediciones de cada uno de los planos antropométricos: frontal, posterior, sagital derecho y sagital izquierdo.

La zona que presenta menor error se encuentra en el plano frontal, tabla 1, con un promedio de error del $1 \%$; mientras que la zona que presenta mayor error está en la tabla 2, con un valor de 3,56\%. Estos errores se pueden deber a las dimensiones de los marcadores ya que el centroide puede variar su posición al realizar cada medición.

Además, los valores que se indican en las tablas 1 y 2 , fueron obtenidos al realizar el promedio de 5 mediciones en cada parte del cuerpo humano, como: hombros, Esternón y otros. Estas mediciones fueron tomadas de los centroides de cada marcador de color blanco. Se toma el centroide de cada marcador y se calculan distancias y ángulos con respecto a estas posiciones.

\begin{tabular}{lcccccc}
\hline Plano Frontal & \multicolumn{7}{c}{ Sujeto uno (1) en cm } & \multicolumn{3}{c}{ Sujeto dos (2) en cm } \\
\hline \multicolumn{1}{c}{ Mediciones } & Sistema & Manual & \%Error & Sistema & Manual & \%Error \\
\hline Hombros & 35.603 & 35,5 & 0,2915 & 34.008 & 34,6 & 1,7104 \\
Esternón & 13.501 & 13,7 & 1,4496 & 14.263 & 14 & 1,879 \\
Brazo derecho & 33.860 & 33,5 & 1,0758 & 32.087 & 32,5 & 1,2708 \\
Antebrazo Derecho & 24.537 & 24 & 2,2383 & 25.751 & 25,2 & 2,1901 \\
Mano Derecha & 20.210 & 19,3 & 4,7171 & 25.751 & 24,4 & 5,5406 \\
Brazo Izquierdo & 34.1182 & 33,8 & 0,9414 & 33.7724 & 33,2 & 1,7241 \\
Antebrazo Izquierdo & 23.1444 & 23 & 0,6278 & 22.2745 & 22,5 & 1,0022 \\
Mano Izquierdo & 22.5261 & 22 & 2,3914 & 23.5381 & 23,2 & 1,4573 \\
Plano Posterior & \multicolumn{2}{c}{ Sujeto uno (1) en cm } & & Sujeto dos (2) en cm \\
\multicolumn{1}{c}{ Mediciones } & Sistema & Manual & \%Error & Sistema & Manual & $\%$ Error \\
Hombros & 35.2013 & 35,5 & 0,8414 & 33.8110 & 33,5678 & 0,7245 \\
Brazo derecho & 32.7984 & 32 & 2,4950 & 34.5192 & 34 & 1,5271 \\
Antebrazo Derecho & 25.9574 & 25,2 & 3,0056 & 24.1621 & 24 & 0,6754 \\
Mano Derecha & 21.6058 & 20,9 & 3,3770 & 22.5413 & 21,9 & 2,9283 \\
Brazo Izquierdo & 32.6514 & 31 & 5,3371 & 34.7521 & 34,2 & 1,6143 \\
Antebrazo Izquierdo & 25.5149 & 24,9 & 2,4695 & 24.5139 & 23,95 & 2,3545 \\
Mano Izquierdo & 18.8691 & 19,2 & 1,7234 & 20.5150 & 20 & 2,5750 \\
Total error & & & 2,7484 & & & 1,7713 \\
\hline
\end{tabular}

Tabla 1. Mediciones Planos frontal y posterior. 


\begin{tabular}{|c|c|c|c|c|c|c|}
\hline \multirow{2}{*}{$\begin{array}{c}\text { Sagital Derecho } \\
\text { Mediciones }\end{array}$} & \multicolumn{3}{|c|}{ Sujeto uno (1) en cm } & \multicolumn{3}{|c|}{ Sujeto dos (2) en cm } \\
\hline & Sistema & Manual & \%Error & Sistema & Manual & \%Error \\
\hline ATM-Cigomático & 8,5567 & 8,2 & 4,3500 & 7,7047 & 7,7 & 0,0610 \\
\hline ATM-Mandíbula & 7,5154 & 7,2 & 4,3806 & 6,3941 & 6,2 & 3,1306 \\
\hline Mandíbula-Gnation & 12,1079 & 11,8 & 2,6093 & 11,5800 & 11,8 & 1,8644 \\
\hline Gnation-ATM & 17,1647 & 16,8 & 2,1708 & 16,6803 & 16 & 4,2519 \\
\hline ATM-Frontal & 16,9789 & 16,5 & 2,9024 & 14,6366 & 16,5 & 11,2933 \\
\hline Brazo derecho & 33,6670 & 32,8 & 2,6433 & 33,6691 & 32,8 & 2,6497 \\
\hline Antebrazo Derecho & 27,0521 & 27 & 0,1930 & 26,3877 & 27 & 2,2678 \\
\hline Mano Derecha & 19,2050 & 19,8 & 3,0051 & 18,9901 & 19,5 & 2,6149 \\
\hline Total error & & & 2,7818 & & & 3,5167 \\
\hline Sagital Izquierdo & \multicolumn{3}{|c|}{ Sujeto uno (1) en cm } & \multicolumn{3}{|c|}{ Sujeto dos (2) en cm } \\
\hline Mediciones & Sistema & Manual & \%Error & Sistema & Manual & \%Error \\
\hline ATM-Cigomático & 8,3654 & 8,2 & 2,0171 & 6,7896 & 6,5 & 4,4554 \\
\hline ATM-Mandíbula & 8,0659 & 7,8 & 3,4090 & 7,4372 & 7,2 & 3,2944 \\
\hline Mandíbula-Gnation & 12,7723 & 12,5 & 2,1784 & 13,5276 & 13,5 & 0,2044 \\
\hline Gnation-ATM & 17,8000 & 17 & 4,7059 & 15,9398 & 15,5 & 2,8374 \\
\hline ATM-Frontal & 17,1256 & 17 & 0,7388 & 14,6075 & 15 & 2,6167 \\
\hline Brazo & 32,6934 & 32 & 2,1669 & 33,6990 & 33,4 & 0,8952 \\
\hline Antebrazo & 26,1159 & 27 & 3,2744 & 24,7245 & 25 & 1,1020 \\
\hline Mano & 20,8655 & 21,1 & 1,1114 & 19,6881 & 20,7 & 4,8884 \\
\hline Total error & & & 2,4502 & & & 2,5367 \\
\hline
\end{tabular}

Tabla 2. Mediciones planos sagital derecho e izquierdo.

Es importante resaltar que las etapas de filtrado y binarización presentan un buen funcionamiento, detectando los marcadores adecuadamente $y$ etiquetándolos de manera correcta. Los resultados de las mediciones anteriores muestran que el sistema funciona con un margen de error de $\pm 3 \%$, usando el error medio cuadrático, lo cual indica que es un algoritmo útil para realizar mediciones antropométricas relacionadas con la acción de la respiración.

Igualmente, los resultados indican que se elaboró una herramienta que a futuro puede servir para realizar seguimientos y búsqueda de patrones característicos en: niños, adolescentes, adultos y adultos mayores y llevar un seguimiento de los diferentes sistemas que intervienen en la evaluación de la respiración.

\section{Conclusiones}

El algoritmo de mediciones antropométricas aplicado para la evaluación de la respiración en tiempo real aquí propuesto, es un sistema de soporte y puede servir como herramienta de ayuda para el especialista en el área de la fonoaudiología. Los resultados mostraron que es posible reducir el tiempo y trabajo de la obtención de un diagnóstico, además de ser mediciones más objetivas que los métodos manuales. Sin embargo, el posicionamiento erróneo de los marcadores es una gran desventaja del sistema: una mala posición tiene como consecuencia malas mediciones. 
El porcentaje deerror en cada zona permite concluir que se debe ajustar la detección de los centroides, debido a que las mediciones presentan un desfase en las distancias de $\pm 1,17 \mathrm{~cm}$. Este resultado hace que sea necesario mejorar el sistema hasta que ese error de distancia se reduzca a cero.

El trabajo a futuro será el de parametrizar variables antropométricas en niños y adultos en diferentes edades, con el fin de obtener variables de normalidades y anormalidad definidas entre las edades. Estas medidas no existen en la literatura actual, medidas que serán los patrones o rangos, normales o no, que permitirían definir si existe alguna situación anormal en los pacientes.

\section{Referencias}

Baek, S., \& Lee, K. (2012). Parametric human body shape modeling framework for human-centered product design. Computer-Aided Design.

Bonilla-González, J. P., \& Prieto-Ortiz, F. A. (2016). Determinación del estado de maduración de frutos de feijoa mediante un sistema de visión por computador utilizando información de color. Revista de Investigación, Desarrollo e Innovación, 7 (1), 111-126. doi: https://doi. org/10.19053/20278306.v7.n1.2016.5603

Bonnet, V., \&Venture, G. (2015). Fast Determination of the Planar Body Segment Inertial Parameters Using Affordable Sensors. IEEE Transactions on Neural Systems and Rehabilitation Engineering, 23 (4), 628-635.

Cárdenas, J. A., \& Prieto-Ortíz, F. A. (2015). Diseño de un algoritmo de corrección automática de posición para el proceso de perforado PCB, empleando técnicas de visión artificial. Revista de Investigación, Desarrollo e Innovación, 5 (2), 107118. doi: $10.19053 / 20278306.3720$

Cerón-Correa, A., Salazar-Jiménez, A. E., \& Prieto-Ortiz, F. A. (2013). Reconocimiento de rostros y gestos faciales mediante un análisis de relevancia con imágenes 3D. Revista de Investigación, Desarrollo e Innovación, 4 (1), 7-20. doi: $10.19053 / 20278306.2563$

Corvalan-Conta, C. (2013). Educación y reeducación postural en niños preescolares. Revista Vinculando. Recuperado de: http://vinculando.org/educacion/ educacion-y-reeducacion-postural-en-ninospreescolares.html

Cruz-Mena, E., \& Moreno-Bolton, R. (2002). Aparato respiratorio Fisiología y clínica. Santiago, Chile: Mediterráneo.

Dixon, F., \& Leonardo, O. (2013) Análisis Cuantitativo De Los Movimientos Articulares Del Cuerpo Humano Y Evaluación De La Postura Global A Nivel De La Columna Vertebral Humana Utilizando Visión Artificial. Pamplona, Colombia: Universidad de Pamplona.

Espitia-Contreras, A., Sanchez-Caiman, P., \& UribeQuevedo, A. (2014). Development of a Kinectbased anthropometric measurement application. IEEE Virtual Reality, 71-72.

Figueredo-Ruiz, J. N., \& Castillo-Martínez, J. A. (2016). Evaluación de desórdenes bucales en profesionales que usan su voz como herramienta de trabajo. Revista Ciencias de la Salud, 14 (especial), 97-112. doi: 10.12804/revsalud14.especial.2016.07

Gelvez-Munevar, P. A., Torres, H. M., MorenoMuñoz, J. C., \& Bautista-Rojas, L. E. (2013). Diseño de un dispositivo electrónico de acciones cíclicas como herramienta de entretenimiento para la inclusión social de personas en estado de cuadriplejía. Revista de Investigación, Desarrollo e Innovación, 4 (1), 21-31. doi:http://dx.doi. org/10.19053/20278306.2604

González, R. C., Woods, R. E., \& Eddins, S. L. (2009). Digital image processing using Matlab. USA: Prentice Hall. 
Gunay, A. (2007), Automatic Detection of Anthropometric Features from Facial Images. Trabzon, Turquía: Karadeniz Teknik University.

Gutiérrez-Ríos, J., Martínez-Oviedo, E. \& PeñaCortés, C. A. (2013). Desarrollo de un módulo didáctico de robótica paralela y visión artificial con un sistema de sujeción universal. Revista Colombiana de Tecnologías de Avanzada, 1 (21), 74 80.

Hernández, B. J., Benjumea, P., \& Tuso, L. F. (2013). Indicadores del desempeño clínico fisioterapéutico en el manejo hospitalario temprano del accidente cerebrovascular (ACV). Revista Ciencias de la Salud, 11 (1), 7-34.

León-Medina, J. X., \& Torres-Barahona, E. A. (2016). Herramienta para el diseño de sistemas de posicionamiento tridimensional usados en fabricación digital. Revista de Investigación, Desarrollo e Innovación, 6 (2), 155-167. doi: http:// doi.org/10.19053/20278306.4603

Orjuela-Juan de Dios, M., Ibarra-González, J. H., Alfonso-Blanco, J., Castro-Chaparro, N. Y., Herrera-Amaya, G. M., \& Valero-Ortíz, A. S. (2015). Caracterización de los resultados de fibrobroncoscópias en el Hospital San Rafael de
Tunja, 2003 a 2012. Revista Investigación en Salud Universidad de Boyacá, 2 (2), 131-147. doi: http:// dx.doi.org/10.24267/23897325.133

Porto de Freitas-Camelo, E. M., Uchôa, D. M., UchoaSantos, F. F., de Vasconcelos, T. B., \& Maia-Macena, R. H. (2015). Use of Softwares for Posture Assessment: Integrative Review 14 (3), 230-235. doi: https:// dx.doi.org/10.1590/S1808-185120151403139932

Susanibar, F. (2014) Protocolo de Evaluación Fonoaudiológica de la Respiración con Puntuación - PEFORP. Perú.

Tsoli, A., Loper, M., \& Black, M. J. (2014), Modelbased anthropometry: Predicting measurements from 3D human scans in multiple poses. Tubingen, Alemania: Max Planck Institute. for Intell.

Vargas, C. E., Ortega, S. J., Leandro, K., Alfonso, M. L., \& Sandoval, C. (2015). Riesgo cardiovascular en la población de 18 a 60 años en la ciudad de Tunja, Colombia. Revista de Investigación, Desarrollo e Innovación, 6 (2), 169-177. doi: http://doi. org/10.19053/20278306.4604

Villar, F., Jareño, J., \& Álvarez, R. (2007) Manual de procedimientos de diagnóstico y control. Madrid, España: Gráficas Enar SA. 